\title{
Imperforate Hymen Causing Hematocolpos and Acute Urinary Retention in a 14-Year-Old Adolescent
}

\author{
Geum Hwa Lee, M.D. ${ }^{1}$, \\ Mi-Jung Lee, M.D. ${ }^{2}$, \\ Young Sik Choi, M.D. ${ }^{3}$, \\ Jae II Shin, M.D. ${ }^{1}$ \\ 'Department of Pediatrics, Severance \\ Children's Hospital, Yonsei University \\ College of Medicine, Seoul, Korea. \\ ${ }^{2}$ Department of Radiology, Severance \\ Hospital, Yonsei University College of \\ Medicine, Seoul, Korea. \\ ${ }^{3}$ Department of Obstetrics and \\ Gynecology, Severance Hospital, \\ Yonsei University College of Medicine, \\ Seoul, Korea.
}

\section{Corresponding author: \\ Jae II Shin, M.D., Ph.D. \\ Address: 50 Yonsei-ro, Seodaemun- \\ gu, Department of Pediatrics, Yonsei \\ University School of Medicine, Seoul 120- \\ 752 , Republic of Korea \\ Tel: +82-2-2228-2050 \\ Fax: +82-2-393-9118 \\ E-mail: shinji@yuhs.ac}

Received: 9 August 2015

Revised: 31 August 2015

Accepted: 13 October 2015
We report the case of a 14-year-old girl who visited the emergency room because of suprapubic discomfort and sudden acute urinary retention. She did not have any significant medical and surgical history, and her neurological examinations were all normal. Urinary catheterization led to the passage of $500 \mathrm{~mL}$ urine. Abdominal ultrasonography showed a hematocolpos that was compressing the urinary bladder. Gynecologic history taking revealed that the patient has not had menarche yet. Therefore, a cruciate incision was performed and her urination became normal. As the surgical outcome after adequate hymenotomy for imperforate hymen is usually good, the diagnosis of imperforate hymen is important. However, this condition is easily missed in the clinic because the first physician visited by the patient rarely takes a detailed gynecologic history or performs appropriate physical examinations. Although rare, imperforate hymen should be considered as a cause of acute urinary retention in the adolescence period. If an adolescent girl presents with abdominal pain and voiding dysfunction, a detailed gynecologic history and appropriate physical examinations of the genital introitus should be performed.

Key words: Imperforate hymen, Hematocolpos, Urinary retention

\section{Introduction}

Imperforate hymen is the most common obstructive anomaly of the female genital tract. It is caused by the noncanalization of the vaginal plate during fetal development ${ }^{1,2}$. The incidence of imperforate hymen is 1 in 1000-10,000 girls, about half of whom will experience urinary retention ${ }^{3)}$. Imperforate hymen mostly occurs sporadically; however, there are several reports of familial cases $^{4}$. It can be diagnosed incidentally during physical examination, or after an evaluation for primary amenorrhea or lower abdominal pain, or rarely, for urinary retention ${ }^{1)}$. We report the case of a 14 -year-old girl who visited the emergency room with sudden acute urinary retention. Hematocolpos was found on abdominal ultrasonography, which led to the diagnosis of imperforate hymen.

\section{Case report}

A 14-year-old girl visited the emergency room because of a sudden onset 

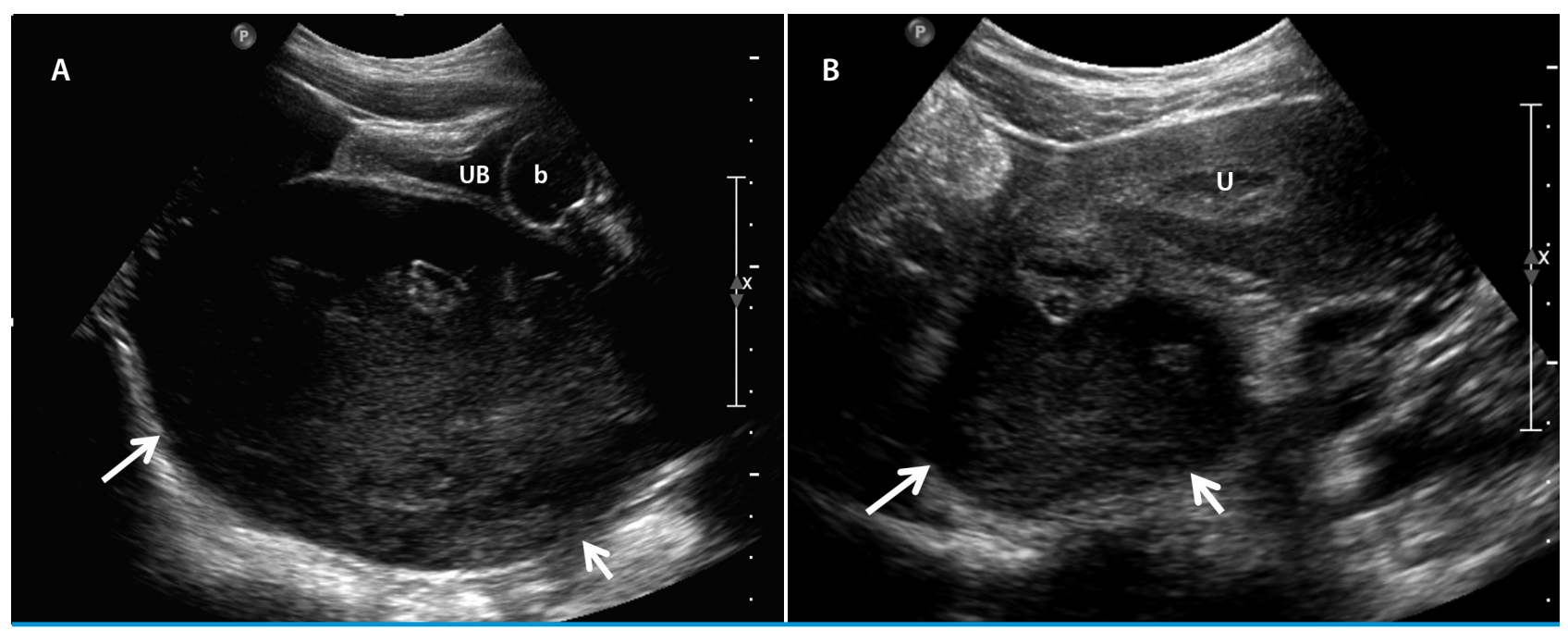

Fig. 1. (A) Sagittal pelvic ultrasonography image showing a huge cystic lesion (arrows) with internal hyperechoic materials compressing the collapsed urinary bladder (UB) anteriorly. The balloon (b) of the Foley catheter in the urinary bladder is also seen. (B) Axial pelvic ultrasonography image of the upper portion of Fig. 1A demonstrating the connection between the huge cystic lesion (arrows) and the displaced uterus $(U)$, suggesting the diagnosis of hematocolpos of the cystic lesion.

of acute urinary retention. She had visited a local clinic for lower abdominal pain, dysuria, frequency, and urgency before she presented to our hospital. She was prescribed oral antibiotics; however, they were not effective. There was no history of abnormal bowel habits. The character of lower abdominal pain was cramping. She did not have any significant medical or surgical history.

On physical examination by a pediatric resident, mild tenderness was noted in the suprapubic area. The neurological examinations were all normal.

The laboratory findings on her first day in our hospital were hemoglobin $12.9 \mathrm{~g} / \mathrm{dL}$, hematocrit $37.0 \%$, white blood cell count $7,650 / \mathrm{mm}^{3}$, and platelet count $263,000 /$ $\mathrm{mm}^{3}$. Albumin level was $4.9 \mathrm{~g} / \mathrm{dL}$ and the other liver function tests were normal. Blood urea nitrogen (BUN) was 9.5 $\mathrm{mg} / \mathrm{dL}$ and creatinine was $0.73 \mathrm{mg} / \mathrm{dL}$. Urinalysis showed a normal result and the urine pregnancy test was negative. Moreover, consultation to the department of urology was done and urinary catheterization was carried out, which led to the relief of her symptoms and the passage of $500 \mathrm{~mL}$ urine.

Abdominal ultrasonography showed a markedly distended vagina with hyperechoic fluid collection compressing the urinary bladder (Fig. 1), which suggested hematocolpos. Further gynecologic history taking revealed that the patient has not had menarche yet, and physical examination on the genital areas confirmed the diagnosis of imperforate hymen. There were no abnormal findings in other organs.

Therefore, according to the ultrasound findings, we consulted the department of gynecology, and hymenotomy by means of a cruciate incision with drainage of the hematocolpos was performed. The patient's postoperative course was good, and the urinary catheter was removed successfully. Her urination became normal, and no further urinary symptom developed.

\section{Discussion}

Imperforate hymen is a rare congenital anomaly of the female genital tract ${ }^{1}$. The hymen usually develops a small opening; however, failure of vaginal plate canalization during fetal development can cause imperforate hymen ${ }^{2}$. Adolescents with imperforate hymen typically present with primary amenorrhea, cyclic lower abdominal or pelvic pain, back pain, constipation, and urinary reten$\operatorname{tion}^{5-7)}$. Acute urinary retention is rare in children ${ }^{8}$; however, the possible causes can include not only neurological diseases but also mechanical obstructions of the urinary tract such as stones, urethral strictures, trauma to the external genitalia, and imperforate hymen ${ }^{8,9}$. Among these, in imperforate hymen, the vagina is filled with menstrual blood (called hematocolpos) ${ }^{10)}$, and subsequently acute urinary retention 
can occur owing to the pressure effect to the bladder and urethra $^{9,11}$.

Therefore, the first menstruation (menarche) can be the first trigger for the development of symptoms due to the accumulation of menstrual blood in the vagina and uterus $^{9)}$. If left untreated, vaginal distension can result in urethral obstruction because of its very close anatomic relation with the anterior vaginal wall ${ }^{2}$. Although rare, it can also present during the neonatal period owing to mucocolpos $^{12)}$.

Theoretically, it may be easy to diagnose imperforate hymen in an adolescent girl with abdominal pain and voiding symptoms, who has not had menarche and shows no introitus in the hymen on physical examination. However, the diagnosis of imperforate hymen is easily missed in the clinic because the first physician rarely takes a detailed gynecologic history or performs appropriate physical examinations ${ }^{1)}$. Therefore, it was reported that imperforate hymen is seldom diagnosed by the physician who first sees the patient ${ }^{13)}$. Similarly, in our case, the first physician in a local clinic did not suspect imperforate hymen, and instead diagnosed cystitis and prescribed oral antibiotics, which were not effective. The next physician in our emergency room did not take a detailed gynecologic history including menarche, and therefore could not suspect imperforate hymen. Even during the insertion of the Foley catheter, the diagnosis of imperforate hymen was not made because of the lack of appropriate physical examinations. The diagnosis of imperforate hymen can be made after an abdominal ultrasonography was done by a radiologist.

The treatment of imperforate hymen is to drain the blood from the vagina and uterus by performing a cruciate incision of the imperforate hymen ${ }^{14)}$. It was emphasized that the uterus should not be squeezed during the course of drainage because it can cause the menstrual blood to flow back through the fallopian tubes into the peritoneal cavity, which may lead to tubal adhesions and endometriosis ${ }^{2,12}$.

The method for the treatment of imperforate hymen is hymenotomy ${ }^{9}$. Although the surgical outcome after adequate hymenotomy for imperforate hymen is usually good, close follow-up is still necessary because some patients experience recurrence or symptoms such as dyspareunia, abnormal menstruation, or persistent problems of micturit- ion or defecation despite an appropriate hymenotomy ${ }^{7}$. However, some authors recommend a hymen-sparing procedure because the integrity of the hymen might be important in some cultures and for some religious groups ${ }^{2)}$.

Although this report is similar to the other reports in general clinic practice ${ }^{15)}$, full physical examination has been ignored in emergency room and in out-patient clinic repeatedly. In our case, the patient firstly visited the outpatient clinic of Endocrinologic department for the evaluation of thyroid function due to suspected goiter a year ago, full history taking and physical examination was not performed at that time. Even once the patient came to the emergency room, the first and the next physician also neglected the physical examination. Importance of physical examination including the hymen should be emphasized in medical education of pediatric residents.

In conclusion, imperforate hymen is a rare and easily missed condition that can cause acute urinary retention in adolescents. Therefore, clinicians should keep in mind that imperforate hymen can be a rare but important cause of acute urinary retention. If an adolescent girl presents with abdominal pain and voiding dysfunction, detailed gynecologic history taking and appropriate physical examinations of the genital introitus should be performed.

\section{Conflict of interest}

No potential conflict of interest relevant to this article was reported.

\section{References}

1. Mou JW, Tang PM, Chan KW, Tam YH, Lee KH. Imperforate hymen: cause of lower abdominal pain in teenage girls. Singapore Med J 2009; 50:378-9.

2. Basaran M, Usal D, Aydemir C. Hymen sparing surgery for imperforate hymen: case reports and review of literature. J Pediatr Adolesc Gynecol 2009; 22:61-4.

3. Lausten-Thomsen MJ, Mogensen H. Hymen imperforatus with atypical symptom presentation. Ugeskr Laeger 2007; 169:523-4.

4. Sakalkale R, Samarakkody U. Familial occurrence of imperforate hymen. J Pediatr Adolesc Gynecol 2005; 18:427-9.

5. Nazir Z, Rizvi RM, Qureshi RN, Khan ZS, Khan Z. Congenital 
www.chikd.org

vaginal obstructions: varied presentation and outcome. Pediatr Surg Int 2006; 22:749-53.

6. Wang W, Chen MH, Yang W, Hwang DL. Imperforate hymen presenting with chronic constipation and lumbago: report of one case. Acta Pediatr Taiwan 2004; 45:340-2.

7. Liang CC, Chang SD, Soong YK. Long-term follow-up of women who underwent surgical correction for imperforate hymen. Arch Gynecol Obstet 2003; 269:5-8.

8. Asgari SA, Mansour Ghanaie M, Simforoosh N, Kajbafzadeh A, Zare' A. Acute urinary retention on children. Urol J 2005; 2:23-7.

9. Abu-Ghanem S, Novoa R, Kaneti J, Rosenberg E. Recurrent urinary retention due to imperforate hymen after hymenotomy failure: a rare case report and review of the literature. Urology 2011; 78:180-2.

10. Deligeoroglou E, lavazzo C, Sofoudis C, Kalampokas T, Creatsas
Lee GH, et al. • Imperforate Hymen Causing Acute Urinary Retention 183

G. Management of hematocolpos in adolescents with transverse vaginal septum. Arch Gynecol Obstet 2012; 285:1083-7.

11. Burgis J. Obstructive Müllerian anomalies: case report, diagnosis, and management. Am J Obstet Gynecol 2001;185:338-44.

12. Sieberg R, Tenhumen A, Yslostatlo OP. Diagnosis of mucocolpos and haematocolpos by ultrasounds; two case reports. J Clin Ultrasound 1985;141:119-28.

13. Tompkins $P$. The treatment of imperforate hymen with hematocolpos. J Am Med Assoc 1939;113:913-6.

14. Bakos O, Berrghind L. Imperforate hymen and ruptured haemosalphinx: a case report with a review of literature. J Adolesc Health 1999;24:226-8.

15. Choi L, Cho SE, Yim HE, Yoo KH, Hong YS, Lee JW. A Case of Imperforate Hymen with Acute Urinary Retention. J Korean Soc Pediatr Nephrol. 2011: 15:86-9. 